\title{
Journal of Surgery Research and Practice
}

Short Communication

Open Access

\section{Subacute Management of Ruptured Lung Hydatid Cysts in Pediatrics} \author{
Al Mohtasib" ${ }^{5}$ Yousef Abu Asbeh ${ }^{6 *}$ \\ ${ }^{1}$ Faculty of Medicine, Al-Quds University, Jerusalem, Palestine \\ ${ }^{2}$ Faculty of Medicine, Al-Quds University, Jerusalem, Palestine \\ ${ }^{3}$ Faculty of Medicine, Al-Quds University, Jerusalem, Palestine \\ ${ }^{4}$ General Surgeon Al-Ahli hospital, Hebron, Palestine \\ ${ }^{5}$ General Surgeon Al-Ahli hospital, Hebron, Palestine
}

Salma Abed Samamqa', Ali Khalid Salah², Shaimaa Awawdeh ${ }^{3}$, Amer Y AbuRumaila ${ }^{4}$, Mohammad Eid

${ }^{6}$ Director of Thoracic Surgery Unit, Al Ahli Hospital, Hebron, Palestine

*Corresponding Author: Yousef Abu Asbeh, Director of Thoracic Surgery Unit, Al Ahli Hospital, Hebron, Palestine; Email: dryousefabuasbeh@gmail.com

Received Date: 18-01-2022; Accepted Date: 11-02-2022; Published Date: 19-02-2022

Copyright $^{\odot} 2022$ by Asbeh YA, et al. All rights reserved. This is an open access article distributed under the terms of the Creative Commons Attribution License, which permits unrestricted use, distribution and reproduction in any medium, provided the original author and source are credited.

\begin{abstract}
Background: The aim of this observational, retrospective study was to review and describe clinical presentations and subacute management of patients with complicated Pulmonary Hydatid Cysts (PHC) and its outcome.

Cases Preview: Six patients, suffering from 10 lung hydatid cysts, with a mean age of 10 years (3-15) year old, of these six patients, there were three males and three females, the most common presentation was ruptured hydatid cyst in four of the cases, all of them were associated with liver hydatid cysts, there were no other organ involvement, the cysts size was ranging from $2.5-10 \mathrm{~cm}$, with mean size of $5 \mathrm{~cm}$.

The presenting symptoms were fever, dry cough, shortness of breath, chest pain on the affected side, hemoptysis, recurrent chest infection, hematemesis and vomiting, we didn't witness any anaphylactic episodes in our study.
\end{abstract}


All the patients were managed surgically after stabilization period range from 2 days- 2 months, there were no intra or post-operative complications, patients were extubated on day 1 post op and the patients were discharged on day 5-8 post-operative on albendazole, with no mortality.

Conclusion: The subacute management of ruptured hydatid cyst results were promising in terms of minimal intra and post-operative complications if presented at all, shorter hospital stay and rapid return to daily activities, early extubation with no need to mechanical ventilation, with no need to rush for an urgent surgery with unstable patient with it's possible complications, this approach was safe, reliable and successful.

\section{Keywords}

Case Series; Rupture; Pulmonary; Hydatid Cyst; Pediatrics; Subacute Management

\section{Introduction}

Hydatid cyst is a zoonotic disease caused by tapeworms from the Echinococcus genus, it is still a common parasitic infestation in the Middle East, where our country located, especially in rural areas where cattle and dogs are kept.

Lung hydatid cysts are more common than liver cysts in children and patients may remain asymptomatic for longer periods due to its higher elasticity considered the most feared complication.

Urgent surgery remains the treatment of choice for ruptured lung hydatid cysts worldwide, followed by a period of medical treatment with albendazole to prevent recurrence.

In this study, we aim to spot the lights on the effectiveness of the newly approached subacute management of ruptured pulmonary hydatid cysts.

To our knowledge, it is the first study to be conducted among pediatric patients in Palestine.

\section{Narrative}

On reviewing 6 patients, who suffered from 10 lung hydatid cysts, with a mean age of 10 years (3-15) year old, of these six patients, there were three males and three females, the most common presentation was ruptured hydatid cyst in 4 of the cases, all of them were associated with liver hydatid cysts, there was no other organ involvement, the cysts size was ranging from 2.5-10 cm, with mean size of $5 \mathrm{~cm}$. In one patient the cysts were bilateral, the hydatid cyst presented in the right lung in two patients and in the left lung in the other three patients. Five 
cysts in the left lung (only one in the upper lobe and four in the lower lobe), 5 cysts were in the right lung ( 3 in the upper lobe, 2 in the lower lobe).

The presenting symptoms were fever, dry cough, shortness of breath, chest pain on the affected side, hemoptysis, recurrent chest infection, hematemesis and vomiting. One of the cases presented with hemodynamic instability due to severe hemoptysis and hematemesis. No patients had skin eruptions, rashes, or anaphylactic episodes.

The diagnosis was made based on clinical presentation and imaging studies mainly CT scan with IV contrast.

Our approach focuses on the subacute surgical intervention, stabilizing the patients before undergoing surgery, four of the presenting cases were started on albendazole for 2 days, one case was maintained on albendazole for one month and another one for 2 months before undergoing elective thoracotomy and hydatid cyst excision.

Pre-op preparations, include routine blood work, including CBC, PT INR, CRP, Kidney Function tests, Liver function tests and serum lytes. Chest imaging with chest X-ray initially followed by Chest CT scan was performed for all the patients.

The surgical approach was performing small VATS incision, in the fifth intercostal space, using the soft tissue retractor disposable wound protector, opening of the adhesions using the Ligasure, identifying the hydatid cyst, then applying multiple gauzes; 5-6 gauzes soaked with hypertonic saline, then applying 22 gauge needle to the hydatid cyst, aspiration of the cyst contents, then injecting of $70 \%$ of the cyst capacity with hypertonic saline $3 \%$, waiting for 20 minutes. Then reaspiration of the cyst content and deroofing of the cyst, using the Ligasure, removal of the germinal layer and starting to perform Cappetonage. Identifying of the bronchial opening of the cyst, applying normal saline and asking the anesthesia to inflate the lung and identifying the leak from the bronchial opening, applying multiple interrupted 0 Vycril stitches, sealing the bronchial opening, then obliterating the whole cyst cavity. Our approach was to avoid resecting healthy lung tissue unless we have to, finally, we perform final check with saline to check for any leak. We leave 20 Fr. Chest tube for 3 days and the patients were extubated immediately post op.

The post-operative course was uncomplicated in all the cases, no mortality. They were all admitted to the ICU one day post operation with placed chest tube, then were transferred to the surgical unit for post operation observation and management including $\mathrm{CBC}$, serial CXR, spirometry and chest physiotherapy. Post op hospital stay ranged from 5 to 8 days.

Patients were all discharged on albendazole for 3-8 months, analgesia and antibiotics. 


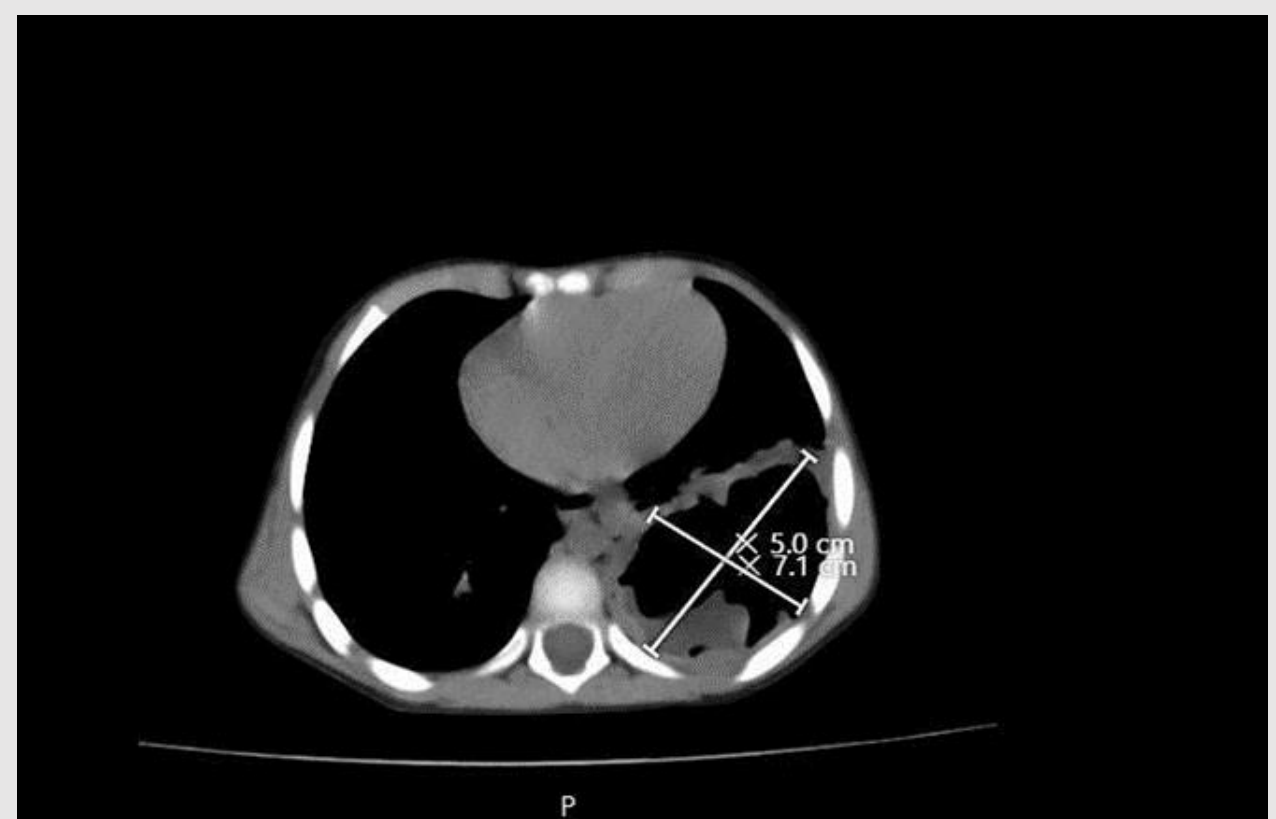

Figure 1: Chest CT scan showing left lower lobe cyst measuring $5 * 7 \mathrm{~cm}$.

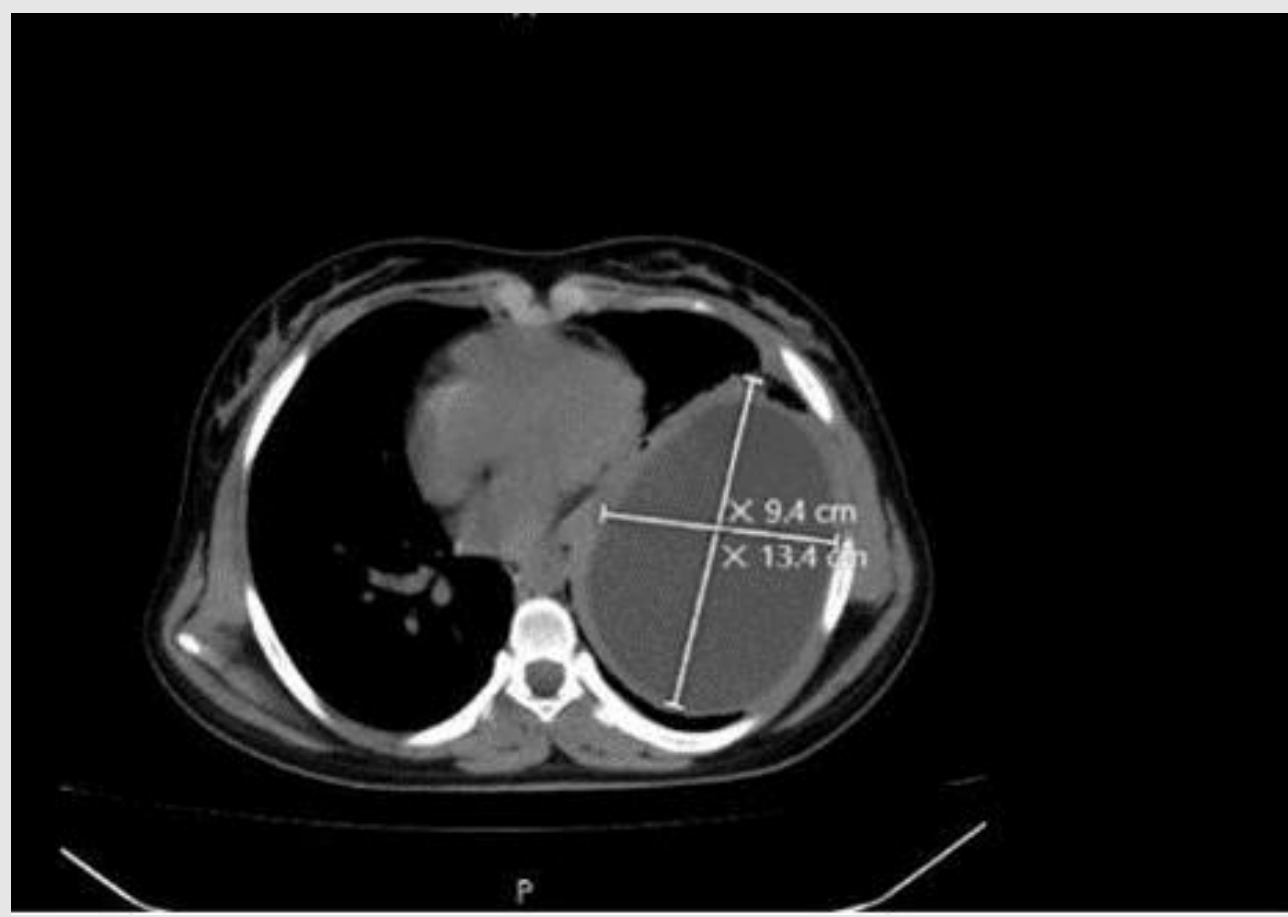

Figure 2: Huge left lung hydatid cyst with surrounding infection. 


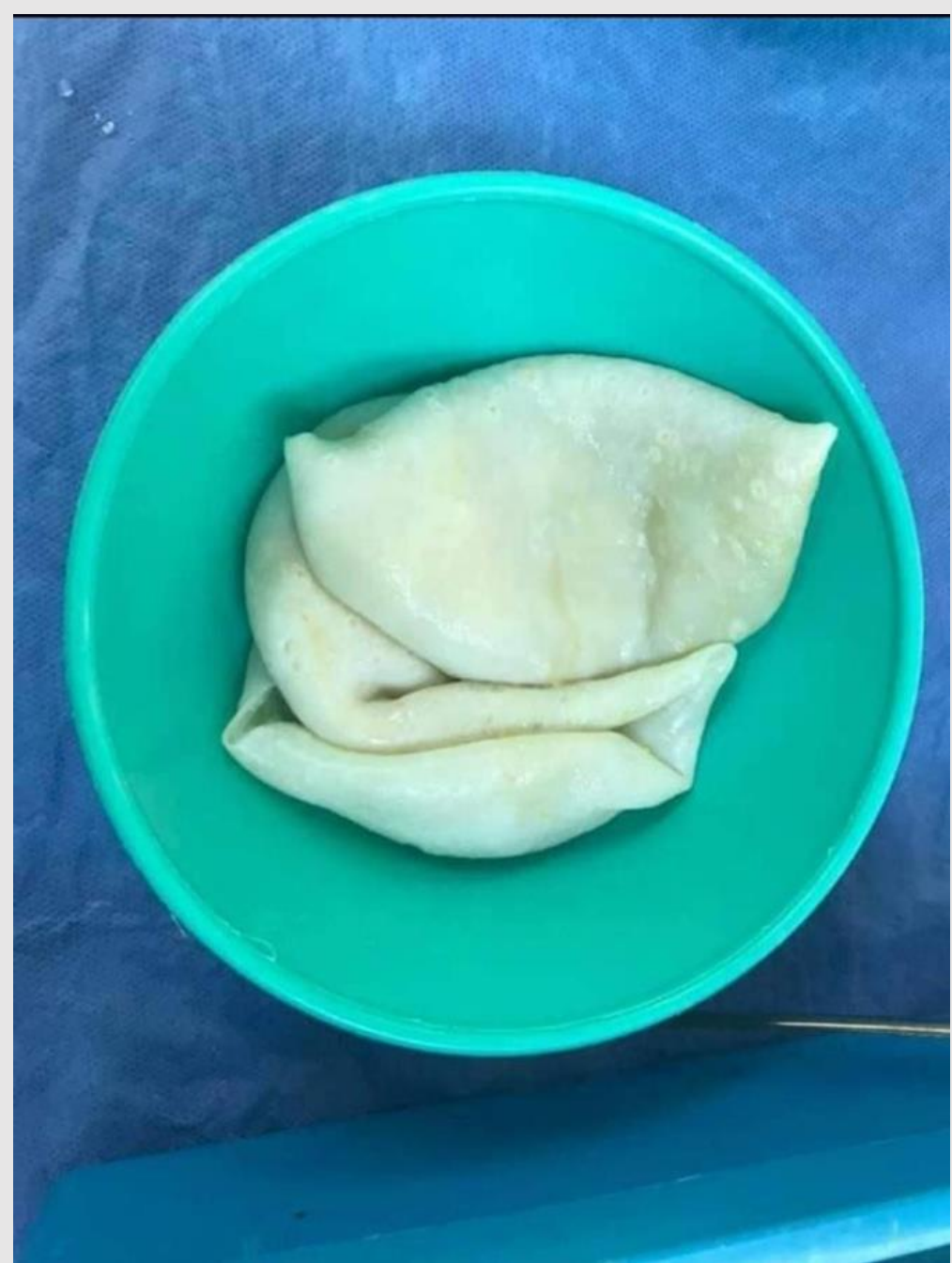

Figure 3: Gross apperance of cyst after excision.

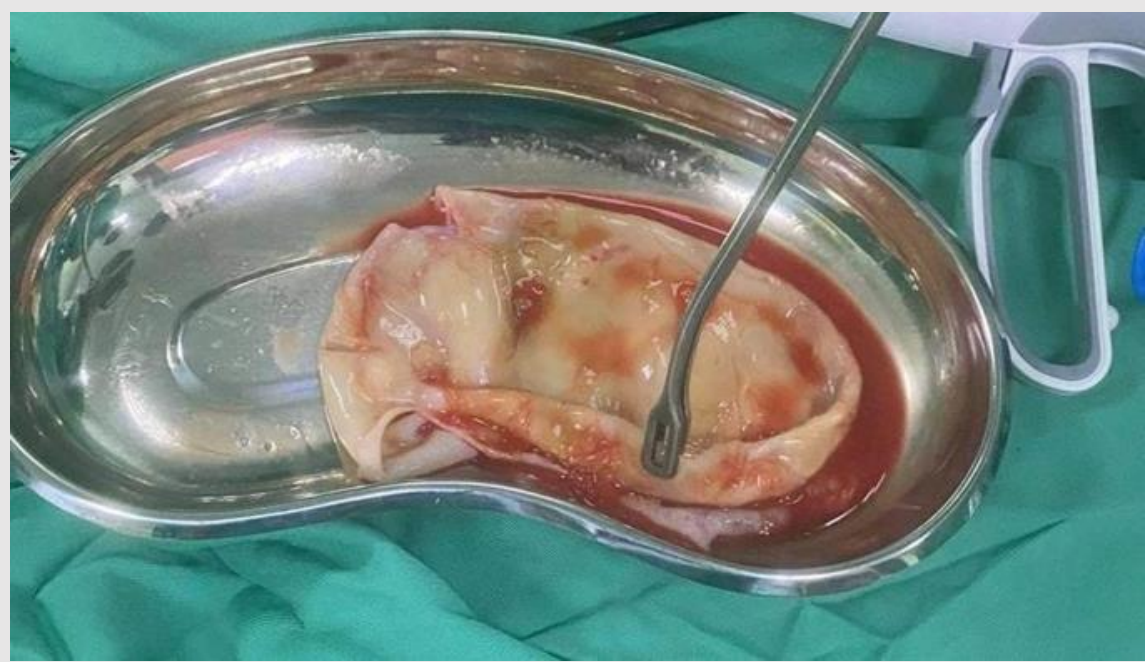

Figure 4: Cyst after excision.

Asbeh YA | Volume 3; Issue 1 (2022) | JSRP-3(1)-031 | Short Communication

Citation: Asbeh YA, et al. Subacute Management of Ruptured Lung Hydatid Cysts in Pediatrics. Surg Res Prac. 2022;3(1):1-7. 


\section{Discussion}

We focused in our study on the subacute management of ruptured hydatid cysts in pediatrics population. we managed the patients medically on albendazole for 2 days- 2 months until they improved clinically, became vitally stable and candidate for the surgery to overcome the morbidity and complications associated with urgent surge defined planes and the extensive adhesions as a result of intense pleural reaction.

The surgeries went smoothly without intraoperative complication.

In H Solak, study was performed on 110 cases all patients were treated surgically; no medical treatment was tried [1-3]. Anatomical resections were needed in 10 cases. And they encountered some complications such as pleural empyema in one case, anaphylactic reaction in 8 cases and one death due to respiratory failure on the third day. Most of the patients $(95 \%)$ have been discharged on the postoperative $8^{\text {th }}$ day.

In Shadi Hamouri study, a similar approach was followed, by administering preoperative albendazole for 4-7 days, all patients except one were treated with a parenchyma sparing resection, the mean hospital stay was 6.9 days [4]. Some complications faced in $16.3 \%$ of the cases including air- leak, broncho-pleural fistula, pneumothorax, without mortality. As well, none of the patients with a ruptured cyst experienced an anaphylactic shock.

Akin Eraslan Balci, recommended that, early surgical intervention with single-lung ventilation andmaximum parenchyma preservation are recommended [5]. The interval between cyst rupture and operation ranged between 24 hours to more than 4 days. Morbidity rate was $25.4 \%$ and mortality was $4.7 \%$.

The strength of this study based on focusing on the pediatric age group.the subacute surgical management.

To our knowledge, it is the first study to be conducted among pediatric patients in Palestine.

\section{The Limitations}

The retrospective nature of this study and the small sample size. Our sample size was 6 , the unicentric approach, the need for longer follow up period.

The urgency of surgical intervention for pulmonary hydatid cyst remains controversial between thetreating physicians, according to our findings in this study, we recommend conducting a multinational, multicenter prospective study to include a larger number of pediatric patients with pulmonary hydatid cysts to investigate the outcome and complications of this approach. 


\section{Conclusion}

The subacute management of ruptured hydatid cyst results were promising in terms of minimal intra and post-operative complications if presented at all, shorter hospital stay and rapid return to daily activities, early extubation with no need to mechanical ventilation, there's no need to rush for an urgent surgery with unstable patient and it's possible complications, this approach was safe, reliable and successful.

\section{Conflict of Interest}

It is stated that there are no conflicts of interest.

\section{References}

1. Manterola C, Otzen T. Surgical strategies in the treatment of liver hydatid cyst. Surgical Practice. 2017;21(4):164-72.

2. Darbari A, Lahiri R, Mishra M, Kumar A, Gautam S, Kumar N. Management of pulmonary hydatid cyst with pleural complications: A case series. European Medical J. 2021.

3. Solak H, Yeniterzi M, Yüksek T, Anil N, Göktoğan T, Ceran S. The hydatid cyst of the lung in children and results of surgical treatment. Thoracic and Cardiovascular Surg. 1990;38(01):45-7.

4. Hamouri S, Odat H, Syaj S, Hecker E, Alrabadi N. Rupture of pulmonary hydatid cyst in pediatrics: A crosssectional study. Annals of Med Surg. 2021;62:31-6.

5. Akin Eraslan Balci, Nesimi Eren, Sevval Eren, Refik Ulkü. Ruptured hydatid cysts of the lung in children: clinical review and results of surgery. Ann Thorac Surg. 2002;74(3):889-92. 